\title{
Solution of the singular Cauchy problem for a general inhomogeneous Euler-Poisson-Darboux equation
}

\section{ELINA SHISHKINA}

\begin{abstract}
.
In this paper, we solve Cauchy problem for a general form of an inhomogeneous Euler-Poisson-Darboux equation, where Bessel operator acts instead of the each second derivative. In the classical formulation, the Cauchy problem for this equation is not correct. However, for a specially selected form of the initial conditions, the equation has a solution. The general form of the Euler-Poisson-Darboux equation with such conditions we will call the singular Cauchy problem.
\end{abstract}

\section{REFERENCES}

[1] Abramowitz, M. and Stegun, I. A., Handbook of Mathematical Functions with Formulas, Graphs, and Mathematical Tables, Applied Mathematics Series, New York, 1983

[2] Barabash, O. P. and Shishkina, E. L., Solution of the general Euler-Poisson-Darboux equation with Bessel operator acting by the all variables, Bulletin of the Tambov University, Series of natural sciences, 6 (2016) 2146-2151

[3] Bateman, H. and Erdélyi, A., Tables of Integral Transforms, Vol. 2. McGraw-Hill, New York, 1954

[4] Blum, E. K., The Euler-Poisson-Darboux equation in the exceptional cases, Proc. Amer. Math. Soc., 5 (1954), 511-520

[5] Bresters, D. W., On the equation of Euler-Poisson-Darboux, SIAM J. Math. Anal., 4 (1973), No. 1, 31-41

[6] Bresters, D. W., On a generalized Euler-Poisson-Darboux equation, SIAM J. Math. Anal., 9 (1978), No. 5, 924-934

[7] Carroll, R. W. and Showalter, R. E., Singular and Degenerate Cauchy problems, Academic Press, New York, 1976

[8] Diaz, J. B. and Weinberger, H. F., A solution of the singular initial value problem for the Euler-Poisson-Darboux equation, Proc. Amer. Math. Soc., 4 (1953), 703-715

[9] Fox, D. N., The solution and Huygens principle for a singular Cauchy problem, J. Math. Mech., 8 (1959), 197-219

[10] Gel'fand, I. M. and Shilov, G. E., Generalized functions. Vol. I: Properties and operations, MA: Academic Press, Boston, 1964

[11] Glushak, A. V. and Pokruchin, O. A., Criterion for the solvability of the Cauchy problem for an abstract EulerPoisson-Darboux equation, Translation of Differ. Uravn., 52 (2016), No. 1, 41?59. Differ. Equ., 52 (2016), No. 1, 39-57

[12] Glushak, A. V., Abstract Euler-Poisson-Darboux equation with nonlocal condition, Russian Mathematics 60 (2016), No. 6, 21-28

[13] Glushak, A. V. and Popova, V. A., Inverse problem for Euler-Poisson-Darboux abstract differential equation, Journal of Mathematical Sciences 149 (2008), No. 4, 1453-1468

[14] Katrakhov, V. V. and Sitnik, S. M., Composition method for constructing B-elliptic, B-hyperbolic, and B-parabolic transformation operators, Russ. Acad. Sci., Dokl. Math., 50 (1995), No. 1, 70-77

[15] Kipriyanov, I. A., Singular Elliptic Boundary Value Problems, Nauka, Moscow, 1997

[16] Levitan, B. M., Expansion in Fourier Series and Integrals with Bessel Functions, Uspehi Matem. Nauk (N.S.) 6 (1951), No. 2 (42), 102-143

[17] Levitan, B. M., Theory of generalised translations operators, Nauka, Moscow, 1973

[18] Lyakhov, L. N., Polovinkin, I. P., and Shishkina, E. L., Formulas for the solution of the Cauchy problem for a singular wave equation with Bessel time operator, Doklady Mathematics, 90 (2014), No. 3, 737-742

Received: 05.05.2017; In revised form: 06.06.2018; Accepted: 13.06.2018

2010 Mathematics Subject Classification. 35L81, 35L15, 58J45.

Key words and phrases. Bessel operator, Euler-Poisson-Darboux equation, Hankel transform. 
[19] Lyakhov, L. N., Polovinkin, I. P., and Shishkina, E. L., On a Kipriyanov problem for a singular ultrahyperbolic equation, Differ. Equ., 50 (2014), No. 4, 513-525

[20] Prudnikov, A. P., Brychkov, Yu. A., and Marichev, O. I., Integrals and Series, Vol. 2, Special Functions, Gordon \& Breach Sci. Publ., New York, 1990

[21] M. Riesz, L'integrale de Riemann-Liouville et le probleme de Cauchy, Acta Mathematica, 81 (1949) 1-223

[22] Shishkina, E. L. and Sitnik, S. M., General form of the Euler-Poisson-Darboux equation and application of the transmutation method, Electron. J. Differential Equations, 177 (2017), 1-20

[23] Shishkina, E. L., Generalized Euler-Poisson-Darboux equation and singular Klein-Gordon equation, Journal of Physics: Conference Series, 973 (2018), No. 1, 1-20

[24] Shishkina, E. L., Singular Cauchy problem for the general Euler-Poisson-Darboux equation, Open Mathematics, 16 (2018), 23-31

[25] Sitnik, S. M., Transmutations and applications: A survey, arXiv:1012.3741v1

[26] Sitnik, S. M., Transmutations and applications, Contemporary studies in mathematical analysis, Vladikavkaz, (2008) 226-293

[27] Sitnik, S. M., Factorization and estimates of the norms of Buschman-Erdelyi operators in weighted Lebesgue spaces, Soviet Mathematics Dokladi, 44 (1992), No. 2, 641-646

[28] Tersenov, S. A., An introduction in the theory of equations degenerating on a boundary, Novosibirsk state university, USSR, 1973

[29] Watson, G. N. A Treatise on the Theory of Bessel Functions, Cambridge University Press, 1922

[30] Weinstein, A., Sur le probléme de Cauchy pour l'équation de Poisson et l'équation des ondes, C. R. Acad. Sci. Paris, 234 (1952), 2584-2585

[31] Weinstein, A., On the wave equation and the equation of Euler-Poisson, Proceedings of Symposia in Applied Mathematics, Vol. V, Wave motion and vibration theory, McGraw-Hill Book Company, Inc., New YorkToronto-London, 137-147, 1954

[32] Weinstein, A., The generalized radiation problem and the Euler-Poisson-Darboux equation, Summa Brasiliensis Mathematicae, 3 (1955), 125-147

[33] Young, F. C., On a generalized EPD equation, J. Math. Mech., 18 (1969), 1167-1175

VORONEZH STATE UNIVERSITY

FACULTY OF APPLIED MATHEMATICS

INFORMATICS AND MECHANICS

UNIVERSITETSKAYA PL., 1, 394000 VORONEZH, RUSSIA

E-mail address: ilina_dico@mail.ru 